\title{
Optimal Machine Tools Selection Using Interval-Valued Data FCM Clustering Algorithm
}

\author{
Yupeng Xin, Xitian Tian, and Lijiang Huang \\ Institute of CAPP \& Manufacturing Engineering Software, Northwestern Polytechnical University, Xian 710072, China \\ Correspondence should be addressed to Lijiang Huang; huanglj@nwpu.edu.cn
}

Received 6 January 2014; Revised 30 April 2014; Accepted 30 April 2014; Published 3 July 2014

Academic Editor: Balaji Raghavan

Copyright (C) 2014 Yupeng Xin et al. This is an open access article distributed under the Creative Commons Attribution License, which permits unrestricted use, distribution, and reproduction in any medium, provided the original work is properly cited.

\begin{abstract}
Machine tool selection directly affects production rates, accuracy, and flexibility. In order to quickly and accurately select the appropriate machine tools in machining process planning, this paper proposes an optimal machine tools selection method based on interval-valued data fuzzy C-means (FCM) clustering algorithm. We define the machining capability meta (MAE) as the smallest unit to describe machining capacity of machine tools and establish MAE library based on the MAE information model. According to the manufacturing process requirements, the MAEs can be queried from MAE library. Subsequently, interval-valued data FCM algorithm is used to select the appropriate machine tools for manufacturing process. Through computing matching degree between manufacturing process machining constraints and MAEs, we get the most appropriate MAEs and the corresponding machine tools. Finally, a case study of an exhaust duct part of the aeroengine is presented to demonstrate the applicability of the proposed method.
\end{abstract}

\section{Introduction}

Machine tools selection is one of the most important strategic decisions in production planning [1]. The proper selection of machine tools can boost manufacturing throughputs and quality of finished products, while reducing manufacturing costs [2]. Machining capacity is one of the main considerations in machine tools selection [3-5]. However, due to the complexity and fuzziness of the machine tools capacity, there are no quantitative criteria to evaluate the matching degree of machine tools and manufacturing process technological requirements. The result is that the machine tool selection scheme made by technologists may be used to focus on individual commonly used, high-performance machines [69]. In this case, it is hard to balance production tasks in the production planning phase, which leads to part of the machine tools' overloaded work, while the other parts of the machine tools are idle and result in a waste of manufacturing resources [10-12].

In order to resolve this problem, Song et al. [13] established machining capacity model of machine tool and obtained feature information by STEP files to inspect machine tools constraints conditions and then to determine whether the machine tools meet the parts machining requirements.
Hao et al. [14] established machining capacity model of machine tool under network collaborative environment and, on this basis, constructed a network manufacturing resource optimization selection system. To resolve matching problem between manufacturing resources and manufacturing process in cross-enterprise process proliferation, Zhao and Mo [15] established machining capacity model of machine tool and resolved matching problem between manufacturing resources and manufacturing process in cross-enterprise process proliferation.

The basic idea of these studies is to take machining capacity as the core to establish machine tool information model, through building the manufacturing process constraints or objective optimization function, and realize machine tools optimal selection using mathematical algorithm. From another perspective, the machine tools selection can be regarded as a matching process of machine tools capacities and manufacturing process technological requirements [16]. However, different machine tools may have similar machining capacities; it is difficult for technologists to estimate which is the most appropriate machine tool for a specific manufacturing process. Therefore, it is necessary to research the machining capacity similarity of different machine tools. Due to the fuzziness of the machine tools 
capacity, researchers try to use fuzzy clustering algorithm to solve this problem.

Tian et al. [17] clustered machining elements using FCM algorithm and optimized clustering analysis by calculating membership degrees of machining methods, cutting tools compatibility, and other process elements. But this method has some difficulties in determining the fuzzy membership and cannot well reflect the matching degrees between machining requirements and machine tools capabilities. In order to solve this problem, Aghdaie et al. [18] calculated the degree of the similarity between the requirements and resources using grey correlation degree and established optimal objective function within the class polymerization degree and separation degree. Aghdaie's work makes up for the deficiency of clustering algorithm proposed in Tian's paper. Liu [19] completed optimal evaluation index system of machine tools and the attribute weighting factor decision algorithm using cluster analysis and rough set theory.

The algorithms described above are only for the single value, however machining capacity model includes intervalvalued data which is hard to be processed by these methods. For solving this problem, Chuang et al. [20] proposed interval-valued data FCM algorithm. On that basis, Ozkan and Türken [21] proposed an improved FCM algorithm for interval-valued data and discussed the relationship of different interval-valued data FCM algorithms. These works have made some progress in interval-valued data fuzzy cluster. The disadvantage is that they still defined distance by Euclidean distance of the point set rather than the distance of interval-valued data. Aiming at this problem, this paper introduces a new distance measure method of interval-valued data and expands FCM clustering algorithm.

In this paper we propose to define the machining capability meta (MAE) as the smallest unit to describe machining capacity of machine tools and quantitatively analyze the machining capacity similarity of different machine tools using a new interval-valued data FCM algorithm. Through computing matching degree between manufacturing process constraints and MAEs, we get the most appropriate MAEs for the manufacturing process and, finally, get the optimal selection of machine tools according to the correspondence between machine tools and MAEs.

The rest of the review paper is synthesized as follows. Section 2 elaborates the definition of MAE and establishes the MAE information model. Section 3 introduces the optimal selection method of machine tools and explains the necessity of interval-valued data FCM algorithm for this method and presents the proposed method in detail. Section 4 presents the case study and,finally, the conclusion is stated in Section 5.

\section{Machining Capacity Meta Model}

Machining capacity elements mainly include machinable parts types, machinable features types, machinable features dimension, machining accuracy, and technical constraints. Among these factors, the machinable features dimension and the machining accuracy are the main metrics of machine tools capacity. In this paper, based on the manufacturing features, we take MAE as the minimum unit to reflect the machining capacity of machine tools, which can be defined as follows.

Definition 1. MAE is the minimum unit to reflect the machine tools machining capacity, its formalized representation is: MAE $:=\{$ ID, BasProperty, EleCapacity $\}$, where ID is the identifier of MAE, BasProperty is the basic property of MAE, EleCapacity is manufacturing meta.

Definition 2. The formalized representation of BasProperty is BasProperty ::= $=\{\mathrm{PT}, \mathrm{FT}, \mathrm{MO}, \mathrm{MT}\}$, where PT is machinable parts types, like axis, sleeve, plate, and box. FT is machinable features types, like hole and groove. MO is machining operation, like turning, milling, and drilling. MT is machinable materials.

Definition 3. Manufacturing meta is the information set of machinable dimension and machining quality. According to different parts types, manufacturing meta contains different elements. One assumes that parts types are divided into rotational and nonrotational, aiming at rotational parts, the formalized representation of manufacturing meta is EleCapacity ::= $\{\mathrm{ML}, \mathrm{MD}, \mathrm{FL}, \mathrm{FD}, \mathrm{MA}, \mathrm{MR}\}$, where $\mathrm{ML}$ is machinable parts length, $\mathrm{MD}$ is machinable parts diameter, FL is machinable feature length, FD is machinable feature diameter, MA is dimensional accuracy, and MR is surface roughness.

According to the above definitions, we establish MAE information model as shown in Figure 1. A MAE corresponds to a manufacturing feature. The manufacturing feature describes the machining region of a machining operation and expresses the result of a machining operation. A machining feature corresponds to a machining operation, which includes machining strategy and machine tool, cutting tools, and fixture. The machining capacity of machine tool is reflected by MAE, a machine tool corresponds to multiple MAEs.

\section{Optimal Machine Tools Selection Method Based on Interval-Valued Data FCM Algorithm}

The optimal selection method proposed in this paper is based on MAE library; the establishment of MAE library needs to summarize the manufacturing features of enterprise's production parts and analyze the machining information of each feature. The machine tools selection process can be divided into preselection phase and optimal selection phase, as shown in Figure 2. In preselection phase, we query the MAEs from MAE library in accordance with the manufacturing process requirements. In optimal selection phase, firstly, using interval-valued data FCM algorithm, we quantitatively analyze the machining capacity similarity of MAEs which were queried in preselection phase and cluster the MAEs into different groups. Secondly, through calculating the matching degree between process constraints and MAEs, we can get 


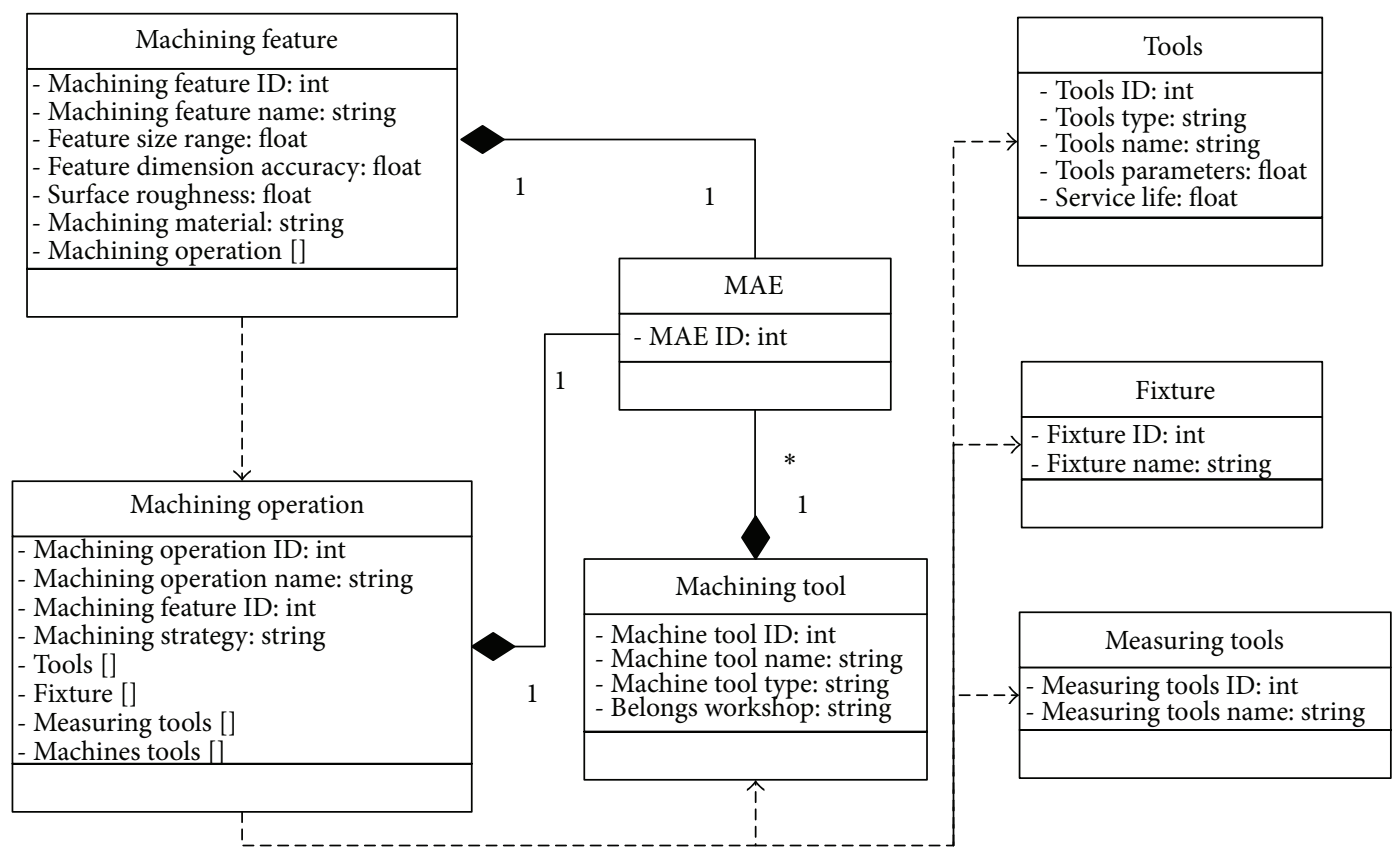

FIgURE 1: The MAE information model.

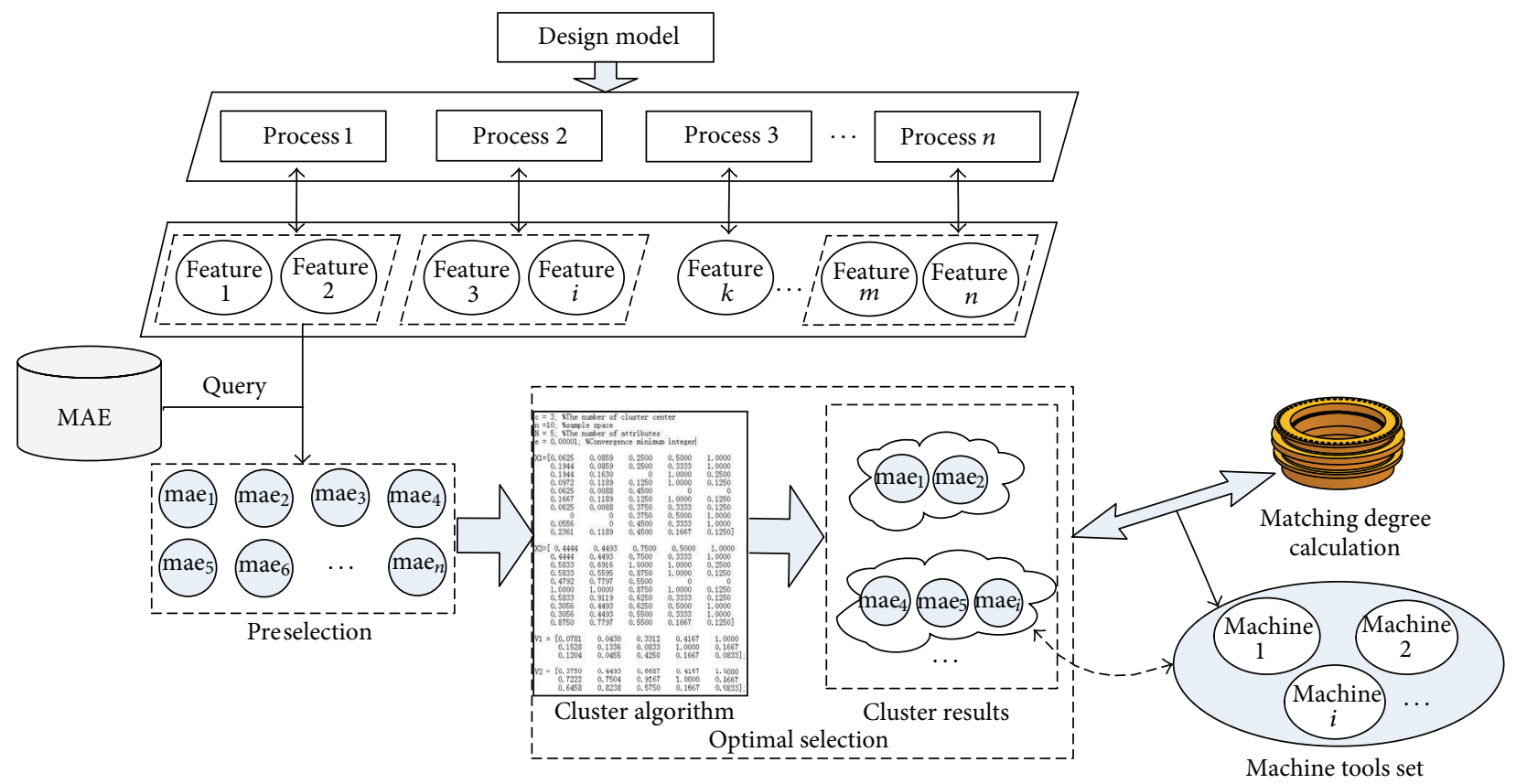

FIGURE 2: The machine tool selection process.

the most appropriate MAEs for the process and, finally, get the most appropriate machine tools according to the responding relationship between MAEs and machine tools.

In preselection phase, considering that BasProperty is nonnumerical, in order to maximum eliminate differences due to inconsistent name and description and insure the accuracy of the matching results, we code BasProperty using parts coding methods as MAEs' ID. Parts coding systems (such as OPTIZ system and KK-3 systems) commonly have a longer code digits [22]; the MAEs coding can simply select part of the codes based on BasProperty.

In optimal selection phase, the MAEs selected in preselection phase is further optimized based on the matching degree of MAEs capacity and manufacturing process requirements; this process needs to solve two problems. (1) How to quantify the similarity of different MAEs machining capacities? (2) How to define and calculate the matching degree between manufacturing process requirements and MAEs? Therefore, 
we focus on the two problems solving method in the following subsection.

3.1. Analysis on MAEs Similarity. In order to quantitatively analyze the machining capacity similarity of MAEs, we build feature space based on EleCapacity. Due to the fact that parts of the EleCapacity elements are interval-valued data (such as machinable part length: $250 \mathrm{~mm} \sim 800 \mathrm{~mm}$ and machinable part diameter: $50 \mathrm{~mm} \sim 120 \mathrm{~mm}$ ), traditional FCM algorithm cannot process such feature space. Therefore, we adopt the distance measure method of interval-valued data proposed by Yu and Fan [23] to calculate fuzzy membership degree of different MAEs and expand the FCM algorithm.

Definition 4. Let $R$ be real number field, for all $a, \bar{a} \in R, \underline{a} \leq \bar{a}$, closed interval data $a=[\underline{a}, \bar{a}]=\{x \mid x \in R, \underline{a} \leq x \leq \bar{a}\}=\{x \mid$ $x=(1-\lambda) \underline{a}+\lambda \bar{a}, \lambda \in[0,1]\}$ is called interval-valued data.

Definition 5. Let $a=[\underline{a}, \bar{a}], b=[\underline{b}, \bar{b}] \in I(R), p \geq 1, x, y \in$ $[0,1]$, and $a_{x}=(1-x) \underline{a}+x \bar{a}, b_{y}=(1-y) \underline{b}+y \bar{b}$, respectively, be the true value of $a$ and $b$. Considering the difference value of each point in $a$ and $b$, we define the distance of $a$ and $b$ as follows:

$$
\begin{aligned}
d(a, b)= & \left(\iint_{0}^{1}\left|a_{x}-b_{y}\right|^{p} d x d y\right)^{1 / p} \\
= & \left(\iint_{0}^{1} \sum_{j=1}^{n} \mid\left[(1-x) \underline{X_{k}^{j}}+x \overline{X_{k}^{j}}\right]\right. \\
& \left.-\left.\left[(1-y) \underline{V_{i}^{j}}+y \overline{V_{i}^{j}}\right]\right|^{P} d x d y\right)^{1 / p} .
\end{aligned}
$$

According to Definition 5, $d(a, b) \geq 0$; if $d(a, b)=0$, then $x=y, d(a, b)=d(b, a)$, one can obtain $d(a, b)$ from Minkowski integral inequality

$$
\begin{aligned}
d(a, b)= & \left(\iint_{0}^{1}\left|a_{x}-b_{y}\right|^{p} d x d y\right)^{1 / p} \\
= & \left(\iint_{0}^{1}\left|\left(a_{x}-c_{z}\right)+\left(c_{z}-b_{y}\right)\right|^{P} d x d y\right)^{1 / p} \\
\leq & \left(\iint_{0}^{1}\left|\left(a_{x}-c_{z}\right)\right|^{P} d x d z\right)^{1 / p} \\
& +\left(\iint_{0}^{1}\left|\left(c_{z}-b_{y}\right)\right|^{P} d z d y\right)^{1 / p} \\
= & d(a, c)+d(c, b),
\end{aligned}
$$

where $c=[\underline{c}, \bar{c}] \in I(R)$, for all $z \in[0,1]$, let $c_{y}=(1-z) \underline{c}+z \bar{c}$ be the true value of $c$. One can also define the distance of interval vectors as follows.
Definition 6. Let interval vectors $A=\left[a_{1}, a_{2}, \ldots, a_{j}, \ldots, a_{n}\right]$ and $B=\left[b_{1}, b_{2}, \ldots, b_{j}, \ldots, b_{n}\right]$, where $a_{j}, b_{j} \in I(R)$, the distance of $A$ and $B$ can be defined as

$$
\begin{aligned}
d(A, B)= & \left(\iint_{0}^{1} \sum_{j=1}^{n}\left|a_{j}-b_{j}\right|^{P} d x d y\right)^{1 / p} \\
= & \left(\iint_{0}^{1} \sum_{j=1}^{n} \mid\left[(1-x) \underline{a_{j}}+x \overline{a_{j}}\right]\right. \\
& \left.-\left.\left[(1-y) \underline{b_{j}}+y \overline{b_{j}}\right]\right|^{P} d x d y\right)^{1 / p}
\end{aligned}
$$

Let feature space be MAE $=\left\{\mathrm{mae}_{1}, \ldots, \mathrm{mae}_{k}, \ldots, \mathrm{mae}_{N}\right\}$, where $\operatorname{mae}_{k}=\left(\operatorname{mae}_{k}^{1}, \ldots, \operatorname{mae}_{k}^{j}, \ldots, \operatorname{mae}_{k}^{n}\right) \in I(R)^{n}, k=$ $1,2, \ldots, N$; $n$ is the number of machining capacity parameters; $c$ is the predefined number of clusters.

The cluster centers matrix is $V^{T}=\left(V_{1}, \ldots, V_{i}, \ldots, V_{c}\right)$, where $V_{i}=\left(V_{i}^{1}, \ldots, V_{i}^{j}, \ldots, V_{i}^{n}\right) \in I(R)^{n}, i=1,2, \ldots, c$.

So one can get fuzzy $c$ division of interval-valued data as follows:

$$
\begin{gathered}
F_{c}=\left\{U_{c \times N}=\left(\mu_{i k}\right)_{c \times N} \in M_{c \times N} \mid \mu_{i k} \in[0,1], \forall i, k ;\right. \\
\left.\sum_{i=1}^{c} \mu_{i k}=1, \forall k ; 0<\sum_{k=1}^{N} \mu_{i k}<N, \forall i\right\},
\end{gathered}
$$

where $i=1,2, \ldots, c, k=1,2, \ldots, N, M_{c \times N}$ is $c \times N$ order matrix set; $\mu_{i k}$ is the membership of the $V_{i}$ cluster; $\sum_{i=1}^{c} \mu_{i k}=$ 1 , for all $k$ indicates that the sum of membership is $1 ; 0<$ $\sum_{k=1}^{N} \mu_{i k}<N$, for all $i$ indicates that each subset is nonempty, neither is universal set $X .0 \leq \sum_{k=1}^{N} \mu_{i k} \leq N$, for all $i$ indicates that empty set and universal set both are allowed; such division space is called degenerate fuzzy $c$ partition space. In cluster analysis, if we can get the optimal division matrix $\left(U_{c \times N}=\left(\mu_{i k}\right)_{c \times N}\right)$ of the specified data set, then the corresponding clusters are the optimal classifications under the specified condition.

The interval-valued data FCM algorithm attempts to minimize the objective function by organizing the data into different clusters. The objective function is as follows:

$$
J_{m}(\operatorname{MAE}, V, c)=\sum_{k=1}^{N} \sum_{i=1}^{c} \mu_{i k}^{m} d_{i k}^{p} \quad(1 \leq m \leq N) \text {, }
$$

where $N$ is the number of data, $c$ is the cluster number, and $i=1,2, \ldots, c, k=1,2, \ldots, N$. 
We can get $d_{i k}$ as follows:

$$
\begin{aligned}
& d_{i k}= d_{i k}\left(\mathrm{MAE}_{k}, V_{i}\right) \\
&=\left(\iint_{0}^{1} \sum_{j=1}^{n} \mid\left[(1-x) \underline{\mathrm{MAE}_{k}^{j}}+\overline{x \mathrm{MAE}_{k}^{j}}\right]\right. \\
&\left.-\left.\left[(1-y) \underline{V_{i}^{j}}+y \overline{V_{i}^{j}}\right]\right|^{P} d x d y\right)^{1 / p},
\end{aligned}
$$

considering $d_{i k} \geq 0$ and $\sum_{j=1}^{n} \mid\left[(1-x) \mathrm{MAE}_{k}^{j}+x \overline{\mathrm{MAE}_{k}^{j}}\right]-$ $\left[(1-y) \underline{V_{i}^{j}}+y{\overline{V_{i}^{j}}}^{P}\right] \mid=0$, let $p=2$, (5) is converted to

$$
\begin{aligned}
J_{m}(\mathrm{MAE}, V, c) & \\
=\sum_{k=1}^{N} \sum_{i=1}^{c} \mu_{i k}^{m} \iint_{0}^{1} \sum_{j=1}^{n} \mid[ & {\left.[1-x) \underline{\mathrm{MAE}_{k}^{j}}+\overline{x \mathrm{MAE}_{k}^{j}}\right] } \\
- & {\left.\left[(1-y) \underline{V_{i}^{j}}+\overline{y V_{i}^{j}}\right]\right|^{2} d x d y . }
\end{aligned}
$$

We can get the iterative solution of (7) as follows:

$$
\begin{gathered}
\mu_{i k}=\left(\sum _ { i = 1 } ^ { c } \left[\iint_{0}^{1} \sum_{j=1}^{n} \mid\left[(1-x) \underline{\mathrm{MAE}_{k}^{j}}+x \overline{\mathrm{MAE}_{k}^{j}}\right]\right.\right. \\
\left.-\left.\left[(1-y) \underline{V_{i}^{j}}+\overline{V_{i}^{j}}\right]\right|^{2} d x d y\right) \\
\times\left(\iint_{0}^{1} \sum_{j=1}^{n} \mid\left[(1-x) \underline{\mathrm{MAE}_{k}^{j}}+x \overline{\mathrm{MAE}_{k}^{j}}\right]\right. \\
-\left[(1-y) \underline{V_{i^{\prime}}^{j}}\right. \\
\left.\left.\left.\left.+y \overline{V_{i^{\prime}}^{j}}\right]\left.\right|^{2} d x d y\right)^{-1}\right]^{1 / m-1}\right)^{-1},
\end{gathered}
$$

where

$$
\underline{V_{i}^{j}}=\overline{V_{i}^{j}}=\frac{\left[\sum_{k=1}^{N} \mu_{i k}^{m}\left(\underline{\mathrm{MAE}_{k}^{j}}+\overline{\mathrm{MAE}_{k}^{j}}\right)\right]}{\left[2 \sum_{k=1}^{N} \mu_{i k}^{m}\right]} .
$$

The selection method of cluster number $c$ refers to reference [24], this paper does not discuss in detail. The FCM algorithm is a kind of local search algorithm $[25,26]$; in order to prevent the results converge to local optimal solution, we need to choose the reasonable initial cluster centers. This paper chooses cluster centers using an improved min-max distance algorithm proposed in reference [27]. The intervalvalued data FCM algorithm can be implemented as follows.
Step 1. Set the predefined number of clusters $c$, where $2 \leq c \leq$ $n, n$ is the number of MAEs.

Step 2. Calculate two MAEs distance $d\left(\mathrm{mae}_{j_{1}}, \mathrm{mae}_{j_{2}}\right), j_{1}, j_{2}=$ $1,2, \ldots, n$. Select the maximum distance of two MAEs which can be expressed as mae $_{p_{1}}$ and mae $_{p_{2}}$. Make mae $p_{p_{1}}$ and $\mathrm{mae}_{p_{2}}$ as the cluster center; $d\left(\mathrm{mae}_{p_{1}}, \mathrm{mae}_{p_{2}}\right) \stackrel{p_{1}}{=}$ $\max _{1 \leq j_{1}, j_{2} \leq n} d\left(\right.$ mae $_{j_{1}}$, mae $\left._{j_{1}}\right)$, where $r=2$.

Step 3. If $c=2$, then go to Step 5, else, calculate the distance of other machining capacity meta mae ${ }_{j}$ and cluster center mae $p_{p_{h}}$ which is expressed as $d\left(\mathrm{mae}_{j}, \mathrm{mae}_{p_{h}}\right), j=1, \ldots, n$ and $\mathrm{mae}_{j} \notin$ $\left\{\operatorname{mae}_{p_{1}}, \ldots\right.$, mae $\left._{p_{r}}\right\}, h=1, \ldots, r$.

If $d\left(\mathrm{mae}_{p_{r+1}}, \operatorname{mae}_{p}\right)=\max \left\{\min _{1 \leq h \leq r} d\left(\mathrm{mae}_{j}, \mathrm{mae}_{p_{h}}\right)\right.$ । $j=1, \ldots, n\}$ and $\mathrm{mae}_{p} \in\left\{\mathrm{mae}_{p_{1}}, \ldots, \mathrm{mae}_{p_{r}}\right\}$, then $\mathrm{mae}_{p_{r+1}}$ is the $(r+1)$ th cluster center; update $r=r+1$.

Step 4. If $r=c$, then go to Step 5; otherwise go to Step 3.

Step 5. Calculate $d\left(\mathrm{mae}_{j}, \mathrm{mae}_{p}\right)=\min _{1 \leq h \leq r} d\left(\mathrm{mae}_{j}, \mathrm{mae}_{p_{h}}\right)$ and classify each MAE based on minimum distance principle. If $j=1,2, \ldots, n$, then put mae ${ }_{j}$ in the group of mae $_{p}$.

Step 6. Calculate the cluster center of each group. Consider:

$$
\begin{aligned}
& v_{0 i}=\left(v_{03}^{i}, v_{04}^{i}, \ldots, v_{0 N}^{i}\right), \quad v_{0 K}^{i}=\left[\underline{v_{0 K}^{i}}, \overline{v_{0 K}^{i}}\right], \\
& \underline{v_{0 K}^{i}}=\frac{1}{N_{i}} \sum_{\mathrm{MAE}_{j} \in G_{i}} A_{k}^{j}, \\
& \overline{v_{0 K}^{i}}=\frac{1}{N_{i}} \sum_{\mathrm{MAE}_{j} \in G_{i}} \overline{A_{k}^{j}} \text {, }
\end{aligned}
$$

where $i=1,2, \ldots, c ; k=3,4, \ldots, N ; j=1,2, \ldots, n ; N_{i}$ is the number of MAEs in the $i$ th group, and $G_{i}$ is the $i$ th group; $V_{0}=\left(v_{01}, v_{02}, \ldots, v_{0 c}\right)$ is the initial cluster center.

Step 7. Standardize the feature space by (11) as follows:

$$
\begin{aligned}
& \overline{A_{k}^{j \prime}}=\frac{A_{k}^{j}-\left(A_{k}\right)_{\min }}{\left(A_{k}\right)_{\max }-\left(A_{k}\right)_{\min }}, \\
& \overline{A_{k}^{j}}=\frac{\overline{A_{k}^{j}}-\left(A_{k}\right)_{\min }}{\left(A_{k}\right)_{\max }-\left(A_{k}\right)_{\min }},
\end{aligned}
$$

where $\left(A_{k}\right)_{\max }=\min _{1 \leq j \leq n}\left\{\overline{A_{k}^{j}}\right\},\left(A_{k}\right)_{\min }=\min _{1 \leq j \leq n}\left\{\underline{A_{k}^{j}}\right\}$, and $k=3,4, \ldots, N$.

For example set parameters $A_{1}^{1}=[200,600], A_{1}^{2}=$ $[750,1000]$, and $A_{1}^{3}=[1500,2000]$, and then we can get $\left(A_{1}\right)_{\max }=2000,\left(A_{1}\right)_{\min }=200$, and $\left(A_{1}\right)_{\max }-\left(A_{1}\right)_{\min }=$ $2000-200=1800$. According to (11) the calculated result of standardized process is $A_{1}^{1^{\prime}}=[0,0.2222], A_{1}^{2^{\prime}}=$ $[0.3056,0.4444]$, and $A_{1}^{3{ }^{\prime}}=[0.7222,1]$.

Step 8. Determine the terminative precision $\varepsilon$, set iterative count number $b=0$. 
Step 9. Calculate the membership $\mu_{i j}{ }^{(b)}(i=1,2, \ldots, c ; j=$ $1,2, \ldots, n)$ by $(8)$.

Step 10. Update each group center $V_{i}^{j}$ and $\overline{V_{i}^{j}}$ by (9).

Step 11. If $\left|J_{m}^{(b+1)}-J_{m}^{(b)}\right|<\varepsilon$, then algorithm ends, and we can get cluster center matrix $V^{*}$ and fuzzy division matrix $U^{*}$; if $\left|J_{m}^{(b+1)}-J_{m}^{(b)}\right| \geq \varepsilon$, set $b=b+1$ and go to Step 9 till the iteration is over.

Fuzzy division matrix $U^{*}$ calculated by FCM algorithm corresponds to the fuzzy division of MAEs. We can make the division more clearly and get general division under two principles as follows.

(1) The principle of minimum distance, which can be elaborated as follows: if $d_{i 0 k}\left(\mathrm{MAE}_{k}-V_{i 0}\right)=$ $\min _{1 \leq i \leq c} d_{i k}\left(\mathrm{MAE}_{k}-V_{i}\right)$, then put $\mathrm{MAE}_{k}$ in the $i_{0}$ th group.

(2) The principle of maximum membership, which can be elaborated as follows: in the $k$ th column of $U^{*}$, if $\mu_{i k}=\max _{1 \leq i \leq c}\left(\mu_{i k}\right)$, then put $\mathrm{MAE}_{k}$ in the $i_{0}$ th group.

\subsection{Matching Degree of Manufacturing Process Requirements} and MAEs. In order to accurately describe the matching degree of manufacturing process requirements and MAEs, this paper defines the concept of EleCapacity matching degree as follows.

Definition 7. EleCapacity matching degree (EMD) reflects the suitability of EleCapacity for manufacturing process requirements. The suitability means that the EleCapacity being either lower or much higher than manufacturing process requirements is inappropriate, which will reduce the matching degree.

The EMD is calculated based on the cluster groups of MAEs. The EleCapacity constraints can be extracted in accordance with manufacturing process requirements. For rotational part, the EleCapacity constraints can be expressed as $R=\{D, L, P A, P R\}$, where $D$ is the outer diameter size range of machining feature, $L$ is the length size range of machining feature, $P A$ is machining accuracy requirements, and $P R$ is surface roughness requirements. The distance between $R$ and cluster centers $V$ reflects the matching degree of manufacturing process requirements and $\mathrm{MAE}_{\mathrm{s}}$, so according to Definition 7 the computational formula of EMD is

$$
\begin{aligned}
\operatorname{EMD} & =d(R, V) \\
& =\left(\iint_{0}^{1} \sum_{i=1}^{c} \sum_{j=1}^{m}\left|r_{j}-v_{i}^{j}\right|^{2} d x d y\right)^{1 / 2}
\end{aligned}
$$

TABLE 1: The verification process technological requirements.

\begin{tabular}{lc}
\hline Property & Parameter \\
\hline Machining method & Turning \\
Machining material & 40CrNiMo \\
Part type & Wheel \\
Feature type & Cylindrical surface \\
Maximum diameter of part $(\mathrm{mm})$ & 550 \\
Maximum length of part $(\mathrm{mm})$ & 150 \\
Outer diameter of feature $(\mathrm{mm})$ & 500 \\
Length of feature $(\mathrm{mm})$ & 100 \\
Machining accuracy $(\mu \mathrm{m})$ & 20 \\
Surface roughness $(\mu \mathrm{m})$ & 1.6 \\
\hline
\end{tabular}

$$
\begin{aligned}
=\left(\iint_{0}^{1} \sum_{i=1}^{c} \sum_{j=1}^{m} \mid[\right. & \left.(1-x) \underline{r_{j}}+x \overline{r_{j}}\right] \\
& \left.-\left.\left[(1-y) \underline{v_{i}^{j}}+y \overline{v_{i}^{j}}\right]\right|^{2} d x d y\right)^{1 / 2},
\end{aligned}
$$

where $c$ is the number of clusters, $m$ is the number of EleCapacity elements, $r_{j}$ is the $j$ th standardized element of EleCapacity process constraints, and $v_{i}^{j}$ is the cluster center of the $i$ th group and the $j$ th element.

According to the minimum distance principle described in Section 3.1, if EMD $=\min _{1<i<c} d_{i}\left(R-V_{i}\right)$, then the $i$ th group is the most appropriate MAEs set to meet manufacturing process requirements.

\section{Case Study}

In this case, we choose a machining example of exhaust duct part of the aeroengine to validate the proposed method in this paper. As shown in Figure 3, after the processes of rough turning exterior surface, rough turning interior surface, turning end face, fine turning exterior surface, fine turning interior surface, and drilling, the forging blank is processed into the exhaust duct part. We choose the process of rough turning exterior surface as verification process and select machine tools for this process using the proposed method.

We summarize the verification process technological requirements as shown in Table 1 and adopt OPTIZ system to encode the part, coding results, and the meaning of each code digit as shown in Figure 4. We select the first five digits and the 7th digit of this part codes to compose MAE's ID; according to Table 1 and OPTIZ system encoding rules, the MAE codes in conformity with the validation process is $0 *$ *302 (* is random number).

Through the matching query in preselection phase, there are 10 MAEs that meet the validation process requirements; the parameters of these MAEs and the corresponding machine tools are presented in Tables 2 and 3. 
TABLE 2: Parameters of MAEs and corresponding machines tools.

\begin{tabular}{|c|c|c|c|c|c|c|}
\hline MAE & MAE codes & $\mathrm{FD}(\mathrm{mm})$ & $\mathrm{FL}(\mathrm{mm})$ & $\mathrm{MA}(\mu \mathrm{m})$ & $\mathrm{MR}(\mu \mathrm{m})$ & Machine tools \\
\hline $\mathrm{mae}_{1}$ & 001302 & {$[250,800]$} & {$[85,250]$} & {$[-10,10]$} & 1 & $m_{1}, m_{2}, m_{3}$ \\
\hline $\mathrm{mae}_{2}$ & 011302 & {$[440,800]$} & {$[85,250]$} & {$[-10,10]$} & 0.8 & $m_{4}$ \\
\hline $\mathrm{mae}_{3}$ & 010302 & {$[440,1000]$} & {$[120,360]$} & {$[-20,20]$} & 1.6 & $m_{5}, m_{6}$ \\
\hline $\mathrm{mae}_{4}$ & 002302 & {$[300,1000]$} & {$[100,300]$} & {$[-15,15]$} & 1.6 & $m_{7}, m_{8}$ \\
\hline $\mathrm{mae}_{5}$ & 013302 & {$[250,850]$} & {$[50,400]$} & {$[-2,2]$} & 0.4 & $m_{9}$ \\
\hline $\mathrm{mae}_{6}$ & 023302 & {$[400,1600]$} & {$[100,500]$} & {$[-15,15]$} & 1.6 & $m_{10}, m_{11}$ \\
\hline $\mathrm{mae}_{7}$ & 021302 & {$[250,1000]$} & {$[50,460]$} & {$[-5,5]$} & 0.8 & $m_{12}, m_{13}, m_{14}$ \\
\hline $\mathrm{mae}_{8}$ & 022302 & {$[160,600]$} & {$[46,250]$} & {$[-5,5]$} & 1.0 & $m_{15}$ \\
\hline $\mathrm{mae}_{9}$ & 012302 & {$[240,600]$} & {$[46,250]$} & {$[-2,2]$} & 0.8 & $m_{16}, m_{17}$ \\
\hline $\mathrm{mae}_{10}$ & 020302 & {$[500,1420]$} & {$[100,400]$} & {$[-2,2]$} & 0.6 & $m_{18}$ \\
\hline
\end{tabular}

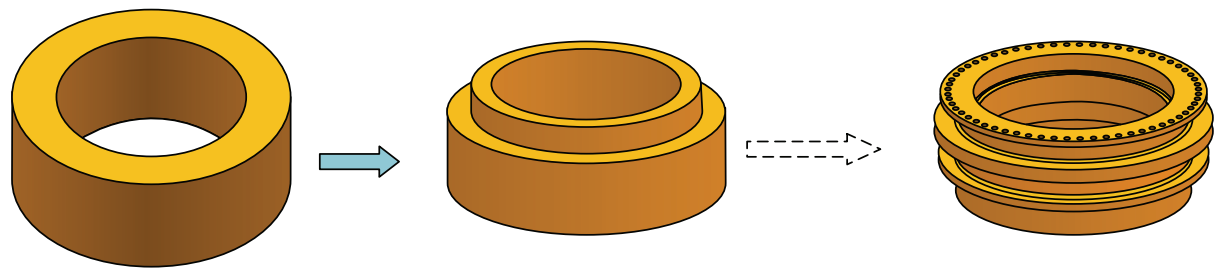

FIGURE 3: The machining process of the exhaust duct part.

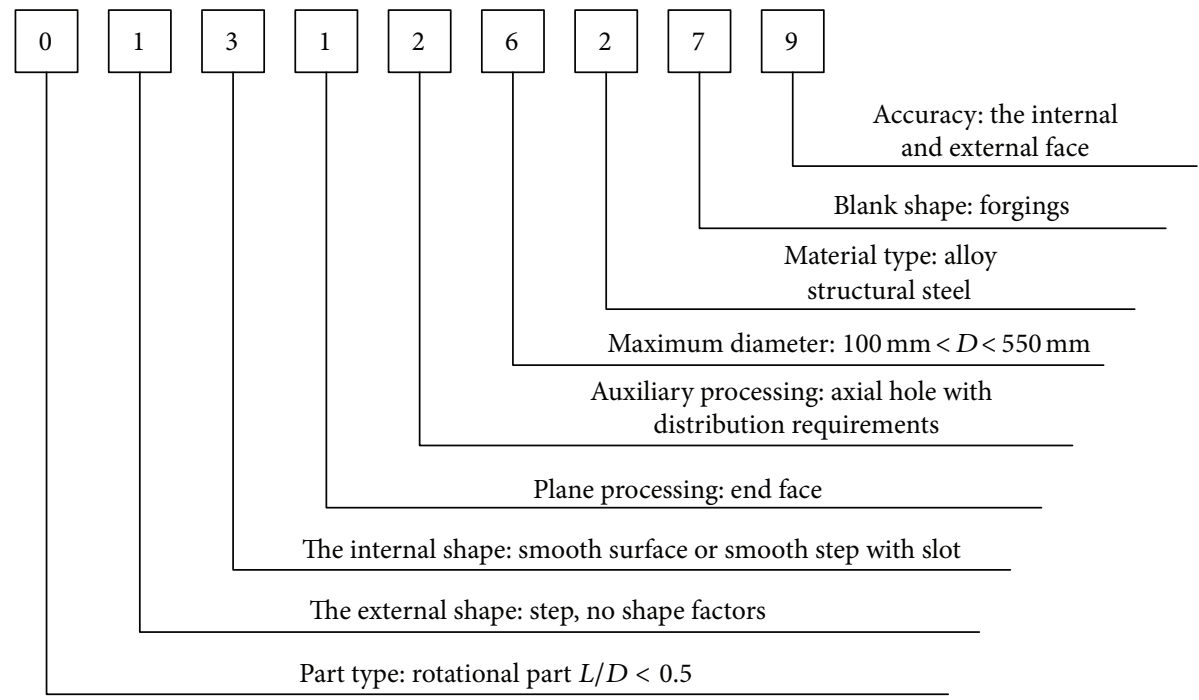

FIGURE 4: Codes information of part.

According to Table 2 we establish feature space and cluster the 10 MAEs using interval-valued data FCM algorithm elaborated in Section 3.1. Let the predefined number of clusters $c=3$ and the terminative precision $\varepsilon=10^{-5}$. Table 4 presents the selection result of the initial cluster center, after 5 iterations using MATLAB simulation; the final calculated results of cluster center and membership are presented in Tables 5 and 6.

We cluster these 10 MAEs in accordance with the maximum membership principle and cluster 18 machines tools as shown in Table 2 based on the correspondence between MAEs and machines tools; the cluster results are shown in Table 7. We can build process constraint vector as $R=$
([500, 550], [100, 150], [-0.02, 0.02], 1.6) and calculate EMD using the method mentioned in Section 3.2: $\mathrm{emd}_{1}=0.0924$, emd $_{2}=0.1271$, and $\mathrm{emd}_{3}=0.2676$. According to the minimum distance principle, the first machines tools set is the most appropriate for this process.

Combining Tables 6 and 7 we can see that MAEs in the same group have a high degree of polymerization (Table 7 shows that the membership degree of MAEs in group 1 has reached more than 0.9); on the other hand, different groups have a high degree of dispersion, which can be reflected by the calculation results of membership degrees as shown in Table 6. 
TABLE 3: The machine tools capacity information.

\begin{tabular}{|c|c|c|c|c|c|c|c|}
\hline $\begin{array}{l}\text { Machine } \\
\text { tool code }\end{array}$ & Machine tool name & Part type & Feature type & $\begin{array}{l}\text { Outer diameter } \\
\text { of feature }(\mathrm{mm})\end{array}$ & $\begin{array}{l}\text { Length of } \\
\text { part }(\mathrm{mm})\end{array}$ & $\begin{array}{c}\text { Machining } \\
\text { accuracy }(\mu \mathrm{m})\end{array}$ & $\begin{array}{c}\text { Surface } \\
\text { roughness (Ra) }\end{array}$ \\
\hline$m_{1}$ & Horizontal CNC lathe 1 & Wheel & Cylindrical surface & $250 \sim 800$ & $85 \sim 250$ & \pm 10 & 1 \\
\hline$m_{2}$ & Horizontal lathe & Wheel & Cylindrical surface & $250 \sim 800$ & $85 \sim 250$ & \pm 10 & 1 \\
\hline$m_{3}$ & Horizontal CNC lathe 2 & Wheel & Cylindrical surface & $250 \sim 800$ & $85 \sim 250$ & \pm 10 & 1 \\
\hline$m_{4}$ & The ground horizontal lathe1 & Wheel & Cylindrical surface & $440 \sim 800$ & $85 \sim 250$ & \pm 10 & 0.8 \\
\hline$m_{5}$ & Vertical lathe 1 & Wheel & Cylindrical surface & $440 \sim 1000$ & $120 \sim 360$ & \pm 20 & 1.6 \\
\hline$m_{6}$ & Single pole vertical lathe & Wheel & Cylindrical surface & $440 \sim 1000$ & $120 \sim 360$ & \pm 20 & 1.6 \\
\hline$m_{7}$ & Vertical lathe 2 & Wheel & Cylindrical surface & $300 \sim 1000$ & $100 \sim 300$ & \pm 15 & 1.6 \\
\hline$m_{8}$ & Double poles vertical lathe & Wheel & Cylindrical surface & $300 \sim 1000$ & $100 \sim 300$ & \pm 15 & 1.6 \\
\hline$m_{9}$ & Ultrafine grinding machine 1 & Wheel & Cylindrical surface & $250 \sim 850$ & $50 \sim 400$ & \pm 2 & 0.4 \\
\hline$m_{10}$ & Vertical CNC lathe 1 & Wheel & Cylindrical surface & $400 \sim 1600$ & $100 \sim 500$ & \pm 15 & 1.6 \\
\hline$m_{11}$ & Vertical CNC lathe 2 & Wheel & Cylindrical surface & $400 \sim 1600$ & $100 \sim 500$ & \pm 15 & 1.6 \\
\hline$m_{12}$ & NC external grinding machine & Wheel & Cylindrical surface & $250 \sim 1000$ & $50 \sim 460$ & \pm 5 & 0.8 \\
\hline$m_{13}$ & Vertical honing machine & Wheel & Cylindrical surface & $250 \sim 1000$ & $50 \sim 460$ & \pm 5 & 0.8 \\
\hline$m_{14}$ & Vertical grinder & Wheel & Cylindrical surface & $250 \sim 1000$ & $50 \sim 460$ & \pm 5 & 0.8 \\
\hline$m_{15}$ & Horizontal CNC lathe 3 & Wheel & Cylindrical surface & $160 \sim 600$ & $46 \sim 250$ & \pm 5 & 1.0 \\
\hline$m_{16}$ & Horizontal CNC lathe 4 & Wheel & Cylindrical surface & $240 \sim 600$ & $46 \sim 250$ & \pm 2 & 0.8 \\
\hline$m_{17}$ & The ground horizontal lathe 2 & Wheel & Cylindrical surface & $240 \sim 600$ & $46 \sim 250$ & \pm 2 & 0.8 \\
\hline$m_{18}$ & Ultrafine grinding machine 2 & Wheel & Cylindrical surface & $500 \sim 1420$ & $100 \sim 400$ & \pm 2 & 0.6 \\
\hline
\end{tabular}

TABLE 4: The parameter values of initial cluster center.

\begin{tabular}{|c|c|c|c|c|c|}
\hline Initial cluster center & $P_{3}$ & $P_{4}$ & $P_{5}$ & $P_{6}$ & $P_{7}$ \\
\hline$v_{01}$ & {$[0.0781,0.3750]$} & {$[0.0430,0.4493]$} & {$[0.3312,0.6687]$} & 0.4167 & 1.0000 \\
\hline$v_{02}$ & {$[0.1528,0.7222]$} & {$[0.1336,0.7504]$} & {$[0.0833,0.9167]$} & 1.0000 & 0.1667 \\
\hline$v_{03}$ & {$[0.1204,0.6458]$} & {$[0.0455,0.8238]$} & {$[0.4250,0.5750]$} & 0.1667 & 0.0833 \\
\hline
\end{tabular}

TABLE 5: The parameter values of final cluster center.

\begin{tabular}{lccccc}
\hline Final cluster center & $P_{3}$ & $P_{4}$ & $P_{5}$ & $P_{6}$ & $P_{7}$ \\
\hline$v_{1}$ & 0.2277 & 0.2484 & 0.5 & 0.4187 & 0.9879 \\
$v_{2}$ & 0.4248 & 0.4325 & 0.5 & 0.9775 & 0.1682 \\
$v_{3}$ & 0.3878 & 0.4328 & 0.5 & 0.1775 & 0.08472 \\
\hline
\end{tabular}

TABle 6: The membership of cluster center.

\begin{tabular}{ccccccccccc}
\hline & $\mathrm{mae}_{1}$ & $\mathrm{mae}_{2}$ & $\mathrm{mae}_{3}$ & $\mathrm{mae}_{4}$ & $\mathrm{mae}_{5}$ & $\mathrm{mae}_{6}$ & $\mathrm{mae}_{7}$ & $\mathrm{mae}_{8}$ & $\mathrm{mae}_{9}$ & $\mathrm{mae}_{10}$ \\
\hline$u_{1 j}$ & 0.9083 & 0.9072 & 0.09448 & 0.06999 & 0.07946 & 0.1046 & 0.1016 & 0.9259 & 0.9426 & 0.07995 \\
$u_{2 j}$ & 0.0461 & 0.04087 & 0.7838 & 0.8224 & 0.09171 & 0.7242 & 0.1748 & 0.0371 & 0.02529 & 0.1098 \\
$u_{3 j}$ & 0.04563 & 0.05191 & 0.1217 & 0.1076 & 0.8288 & 0.1712 & 0.7236 & 0.03699 & 0.03211 & 0.8102 \\
\hline
\end{tabular}

TABLE 7: The cluster results of machines tools.

\begin{tabular}{lcc}
\hline Group & MAE set & Machines tools set \\
\hline 1 & $\mathrm{mae}_{1}, \mathrm{mae}_{2}, \mathrm{mae}_{8}, \mathrm{mae}_{9}$ & $m_{1}, m_{2}, m_{3}, m_{4}, m_{15}, m_{16}, m_{17}$ \\
2 & $\mathrm{mae}_{3}, \mathrm{mae}_{4}, \mathrm{mae}_{6}$ & $m_{5}, m_{6}, m_{7}, m_{8}, m_{10}, m_{11}$ \\
3 & $\mathrm{mae}_{5}, \mathrm{mae}_{7}, \mathrm{mae}_{10}$ & $m_{9}, m_{12}, m_{13}, m_{14}, m_{18}$ \\
\hline
\end{tabular}


In Table 7, $\mathrm{mae}_{7}$ and $\mathrm{mae}_{8}$ have the same machining accuracy (the values of MA are equal), but they belong to different groups; it proves that the cluster results depend on the coupling effect of the whole machining capacity parameters, and not on being restricted by a single parameter.

The algorithm has only 5 iteration times in computational experiment and can rapidly converge to the preset minimum value. Therefore, the computational efficiency of this algorithm is relatively high.

\section{Conclusion}

Because of the complexity and fuzziness of machine tools capacity, it is difficult to evaluate machine tools matching degree for manufacturing process. In this research, we define MAE to describe the machine tools capacity. Based on the MAE information model, we propose a machine tools optimal selection method using interval-valued data FCM clustering algorithm. Finally, we get the most appropriate machine tools by the EMD which reflects matching degree of machine tools and manufacturing process. Aiming at the interval-valued data in MAE model, we have expanded the traditional FCM algorithm.

The results show that the proposed interval-valued data FCM clustering algorithm can cluster feature space, lessen impact of individual parameter on the clustering results, and ensure the objectivity of the clustering results. The optimal machine tools selection method proposed in this research can help technologists to choose the appropriate machine tools for manufacturing process in machining process planning.

\section{Conflict of Interests}

The authors declare that there is no conflict of interests regarding the publication of this paper.

\section{References}

[1] S. Perçin and H. Min, "Optimal machine tools selection using quality function deployment and fuzzy multiple objective decision making approach," Journal of Intelligent and Fuzzy Systems, vol. 24, no. 1, pp. 163-174, 2013.

[2] Y. Zhang, P. Jiang, G. Q. Huang, T. Qu, and J. Hong, “Taskdriven e-manufacturing resource configurable model," Journal of Intelligent Manufacturing, vol. 23, no. 5, pp. 1681-1694, 2012.

[3] A. Kusiak, Computational Intelligence in Design and Manufacturing, John Wiley \& Sons, New York, NY, USA, 2000.

[4] M. G. Mehrabi, A. G. Ulsoy, and Y. Koren, "Reconfigurable manufacturing systems: key to future manufacturing," Journal of Intelligent Manufacturing, vol. 11, no. 4, pp. 403-419, 2000.

[5] L. N. Zhu, Y. W. Zhao, W. L. Wang et al., "A bilayer resource model for cloud manufacturing services," Mathematical Problems in Engineering, vol. 2013, Article ID 607582, 10 pages, 2013.

[6] H. F. de Castro and K. L. Cavalca, "Maintenance resources optimization applied to a manufacturing system," Reliability Engineering and System Safety, vol. 91, no. 4, pp. 413-420, 2006.

[7] M. Ulieru and D. Norrie, "Fault recovery in distributed manufacturing systems by emergent holonic re-configuration: a fuzzy multi-agent modeling approach," Information sciences, vol. 127, no. 3-4, pp. 101-123, 2000.

[8] H. Chen, A. Wang, and R. Ning, "Modularized cluster analysis during manufacturing cell formation," Journal of Huazhong University of Science and Technology (Natural Science Edition), vol. 40, no. 2, pp. 87-90, 2012.

[9] M. Sheikhzadeh, S. Benjaafar, and D. Gupta, "Machine sharing in manufacturing systems: total flexibility versus chaining," International Journal of Flexible Manufacturing Systems, vol. 10, no. 4, pp. 351-378, 1998.

[10] S. Reveliotis, E. Roszkowska, and J. Y. Choi, "Correctness verification of generalized algebraic deadlock avoidance policies through mathematical programming," IEEE Transactions on Automation Science and Engineering, vol. 7, no. 2, pp. 240-248, 2010.

[11] A. Karnik, C. S. Tallichetty, and A. Saroop, "Robust models for manufacturing capacity planning under demand uncertainty," in Proceedings of the IEEE International Conference on Automation Science and Engineering (CASE'09), pp. 310-315, Bangalore, India, August 2009.

[12] D. Corti, A. Pozzetti, and M. Zorzini, "A capacity-driven approach to establish reliable due dates in a MTO environment," International Journal of Production Economics, vol. 104, no. 2, pp. 536-554, 2006.

[13] Y. Y. Song, X. P. Chu, and F. Z. Cai, "Research on manufacturing resource capability modeling and its application based on STEP," Computer Integrated Manufacturing Systems, vol. 5, no. 4, pp. 46-50, 1999.

[14] J. H. Hao, S. D. Sun, and Q. Y. Sha, "Resource modeling oriented generalized manufacturing capability," Journal of Computer Applications, no. 3, pp. 60-63, 2006.

[15] Y. Zhao and R. Mo, "Matching capability degree of manufacturing resource capability and manufacturing process constraint," Computer Integrated Manufacturing Systems, vol. 15, no. 4, pp. 712-718, 2009.

[16] F. Liang, R. Y. K. Fung, and Z. Jiang, "Modelling approach and behaviour analysis of manufacturing resources in virtual cellular manufacturing systems using resource element concept," International Journal of Computer Integrated Manufacturing, vol. 24, no. 12, pp. 1168-1182, 2011.

[17] F. J. Tian, X. T. Tian, J. H. Geng, and Z. Zhang, "Process planning method driven by process model," Computer Integrated Manufacturing Systems, vol. 17, no. 6, pp. 1128-1134, 2011.

[18] M. H. Aghdaie, S. H. Zolfani, and E. K. Zavadskas, "Decision making in machine tool selection: an integrated approach with SWARA and COPRAS-G methods," Inzinerine Ekonomika, vol. 24, no. 1, pp. 5-17, 2013.

[19] G. Q. Liu, "Decision-making on optimal resource allocation in regional virtual enterprises alliance based on RST," Computer Integrated Manufacturing Systems, vol. 13, no. 11, pp. 2178-2183, 2007.

[20] C. C. Chuang, J. T. Jeng, and C. W. Li, "Fuzzy c-means clustering algorithm with unknown number of clusters for symbolic interval data," in Proceedings of the SICE Annual Conference, pp. 358-363, Tokyo, Japan, 2008.

[21] I. Ozkan and I. B. Türken, "MiniMax $\varepsilon$-stable cluster validity index for type-2 fuzziness," Information Sciences, vol. 184, no. 1, pp. 64-74, 2012.

[22] Z. M. Zhang, J. X. Xu, and X. L. Jia, Modern CAPP Technology and Application, Northwestern Polytechnical University Press, Xi'an, China, 2004. 
[23] C. H. Yu and Z. P. Fan, "A clustering method based on interval numbers for multi-index information," Journal of Northeastern University: Natural Science, vol. 25, no. 2, pp. 183-186, 2004.

[24] S. B. Zhou, Z. Y. Xu, and X. Q. Tang, "New method for determining optimal number of clusters in K-means clustering algorithm," Computer Engineering and Application, vol. 46, no. 16, pp. 27-31, 2010.

[25] Y. Zhang, P. Li, Y. Wang, P. Ma, and X. Su, "Multiattribute decision making based on entropy under interval-valued intuitionistic fuzzy environment," Mathematical Problems in Engineering, vol. 2013, Article ID 526871, 8 pages, 2013.

[26] Z. Zhang, M. Wang, Y. Hu, J. Yang, Y. Ye, and Y. Li, "A dynamic interval-valued intuitionistic fuzzy sets applied to pattern recognition," Mathematical Problems in Engineering, vol. 2013, Article ID 408012, 10 pages, 2013.

[27] F. M. Sleim and H. I. Mustafa, "On interval-valued supra-fuzzy syntopogenous structure," Mathematical Problems in Engineering, vol. 2012, Article ID 854942, 8 pages, 2012. 


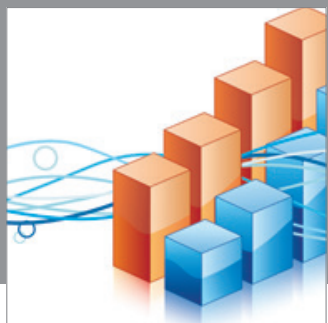

Advances in

Operations Research

mansans

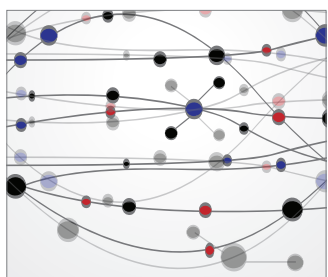

The Scientific World Journal
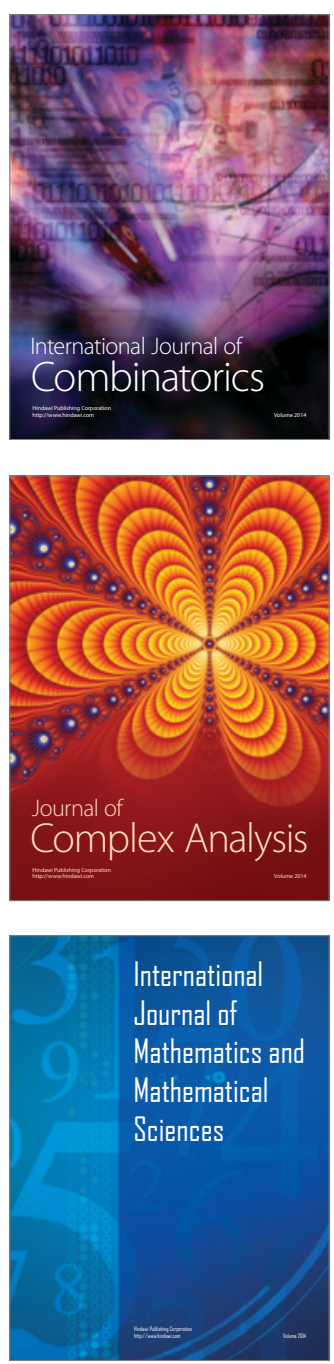
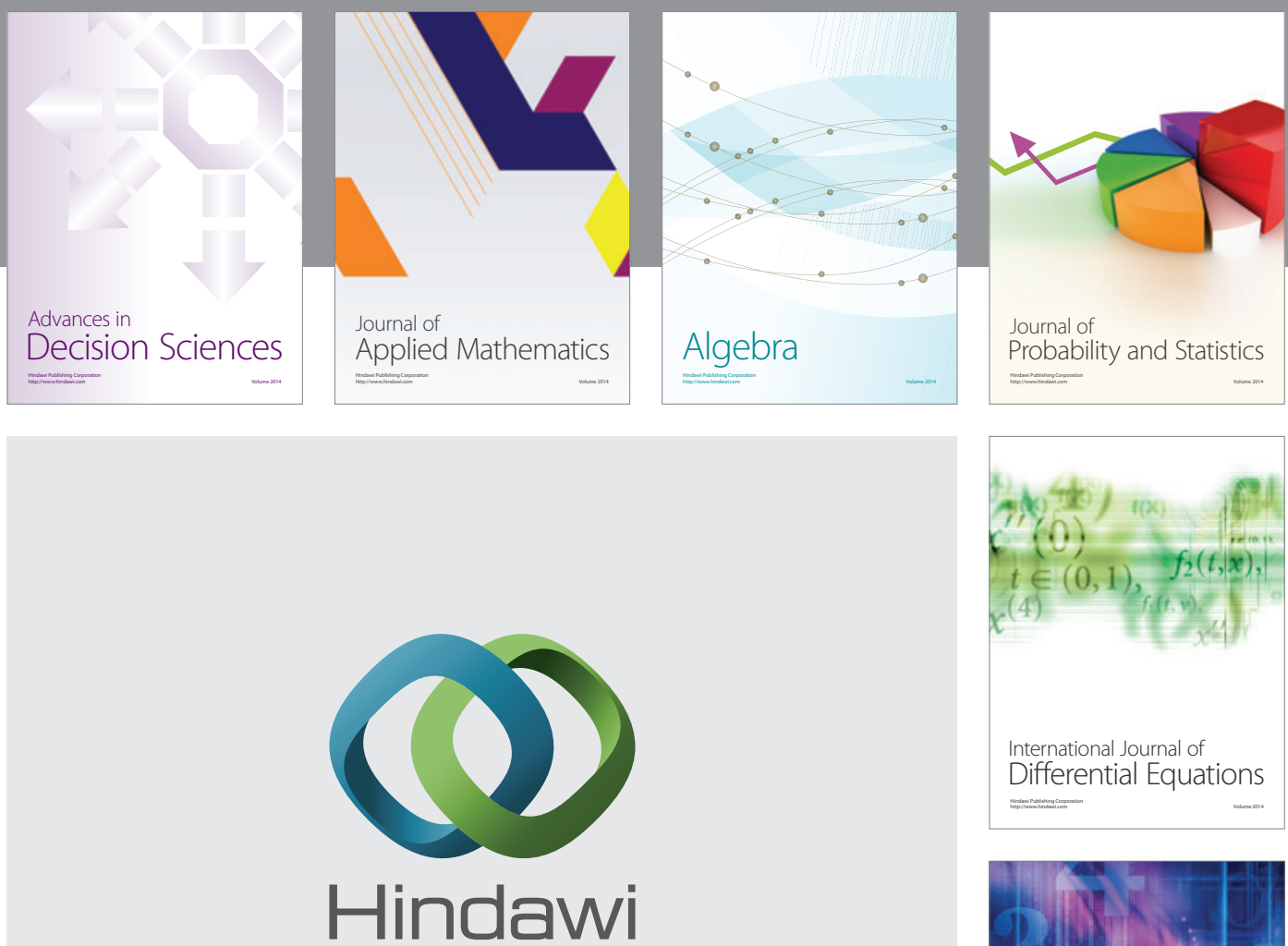

Submit your manuscripts at http://www.hindawi.com
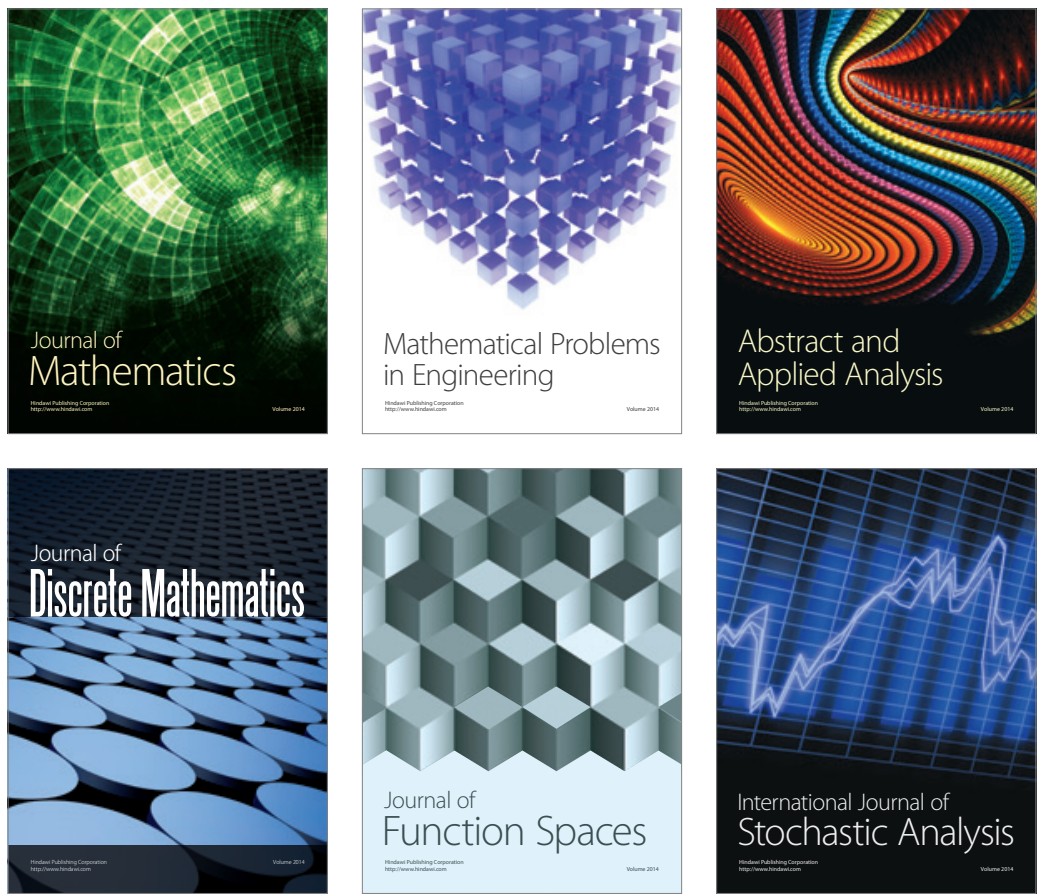

Journal of

Function Spaces

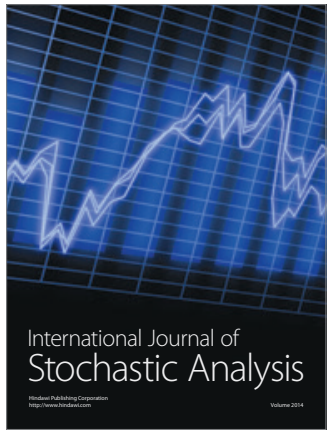

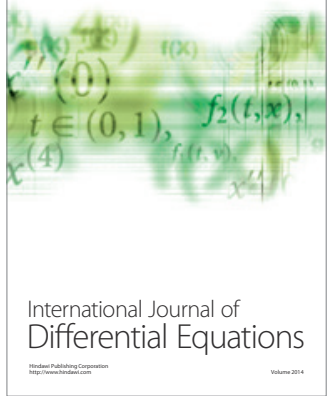
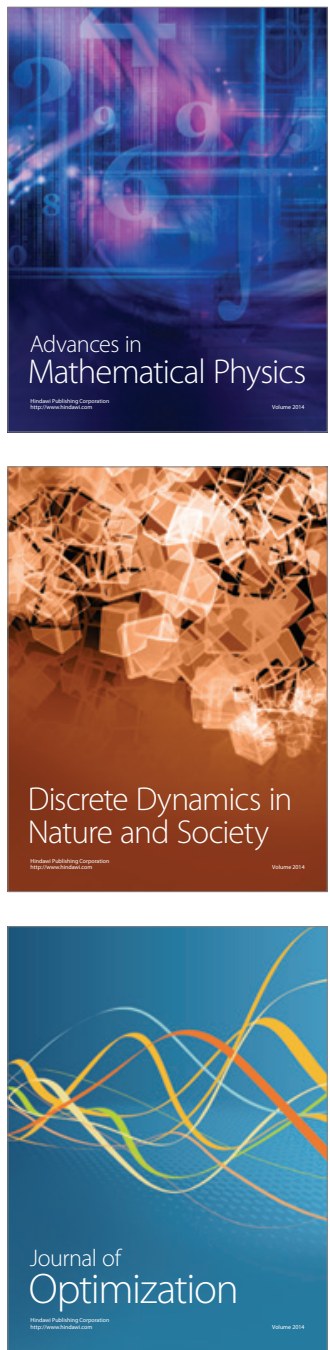